
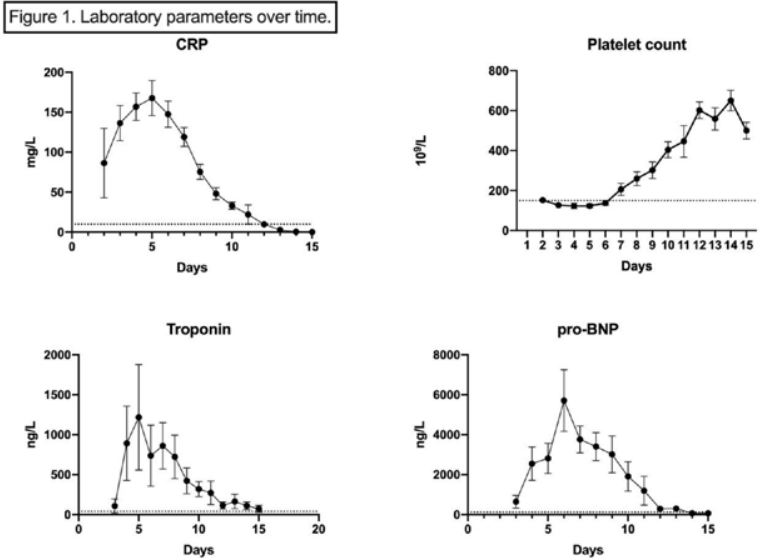

LDH
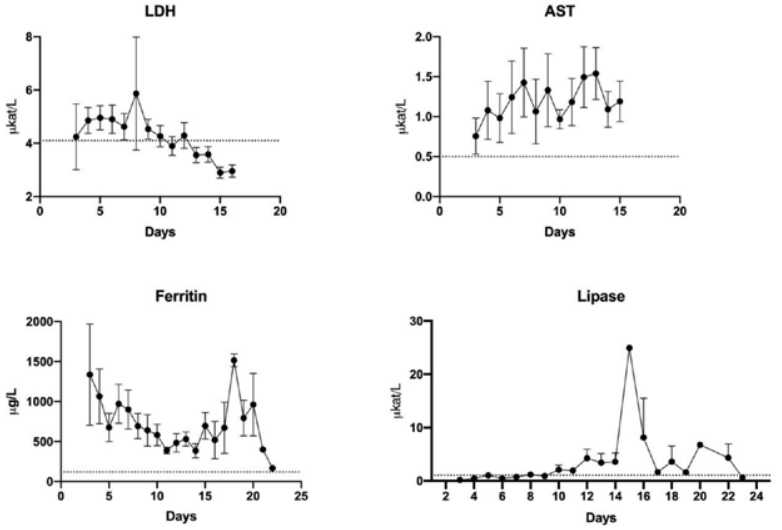

Normal values are shown in dotted line. Lipase is shown only in patients with at least one pathological value.

Conclusion: A very high incidence of MIS-C, estimated 5.8/100 000 persons under the age of 19 with a predominantly cardiac involvement but very good outcome was noted in European Caucasian population in a nationwide cohort study in Slovenia. Attention to newly described pancreatic involvement should be raised.

Disclosure of Interests: None declared

DOI: 10.1136/annrheumdis-2021-eular.3022

\section{POS1319 RAYNAUD'S PHENOMENON IN A SINGLE CENTER COHORT OF TURKISH CHILDREN}

S. Çağlayan ${ }^{1}$, F. Demir ${ }^{1}$, B. Sancar ${ }^{1}$, K. Ulu' ${ }^{1}$, T. Coşkuner ${ }^{1}$, B. Sözeri' ${ }^{1}{ }^{1}$ Health Sciences University, Umraniye Traning and Research Hospita, Pediatric Rheumatology, Istanbul, Turkey

Background: Raynaud's phenomenon (RF) is a vasospastic condition characterized by episodic color changes of blanching, cyanosis, and hyperemia of the extremities of the body

Objectives: We aim to examine the clinical presentation, capillaroscopic findings and disease associations of Raynaud's phenomenon (RP) in pediatric patients Methods: We retrospectively enrolled a single-center cohort of 247 consecutive pediatric patients with RP admitted to Umraniye Training and Research Hospital, Pediatric Rheumatology Clinic, Istanbul, Turkey, since 2016. Medical records were analyzed for clinical presentation, disease associations, and physical examination and laboratory findings.

Results: We reported 247 patients (152 female, 95 male) with RP. Their mean age at disease onset was $14.5 \pm 2.55$ years and median age at diagnosis was $15.4 \pm 2.21$ years. In patients with secondary RP syndrome $(10.9 \%)$, the age of complaint was found to be lower than others $(p=0.03)$. Although most patients have affected upper extremity (hand and fingers), lower extremity (toes) involvement was also detected in $50(20 \%)$ patients. In both the patients with primary and secondary RP, main triggering factor was found as cold exposure. Biphasic (53.6\%) color changes were more seen in primary $\mathrm{RP}$, although triphasic $(51.8 \%)$ color changes were more in patients with secondary RP $(p=0.00)$. The presence of antinuclear antibodies and abnormal nailfold capillaries were more seen in patients with secondary RP (28\% vs $74 \%$ and $35.3 \%$ vs 66.7 , respectively $p=0.00$ ). Digital ulcer was detected $5.2 \%$ of patients (mostly secondary). Out of 247,31 patients were treated with lowdose aspirin, 47 with calcium blockers, 25 with low-dose aspirin and calcium blockers, 16 with iloprost and/or bosentan, while the remaining 140 did not receive any drug.

Conclusion: Similar to adults, RP is more common in girls without an underlying disease. In patients with secondary RP, the symptoms. It has been shown that in patients with secondary RP, symptoms begin at a younger age and the ANA positivity and abnormal nailfold capillaries correlate.

REFERENCES:

[1] Jones G, et al. Arthritis Rheum 2003;48:3518-352

[2] Garner R et al. BMJ Open. 2015; 5(3): e006389

[3] Nigrovic PA et al. Pediatrics 2003, 111 (4) 715-721

Disclosure of Interests: None declared

DOI: 10.1136/annrheumdis-2021-eular.3076

\section{POS1320 DIFFERENCES IN CLINICAL MANIFESTATION AND DISEASE ACTIVITY OF PEDIATRIC BEHÇET DISEASE: A CROSS-SECTIONAL COHORT COMPARISON BETWEEN TURKEY AND UNITED STATES}

E. Demirkaya ${ }^{1}$, M. Romano ${ }^{1}$, C. Swearingen ${ }^{2}$, O. Kasapcopur ${ }^{3}$, B. Makay ${ }^{4}$, S. Özen ${ }^{5}$, Y. Yazici ${ }^{6} .{ }^{1}$ Schulich School of Medicine \& Dentistry, University of Western Ontario, Paediatric Rheumatology, LONDON, Canada; ${ }^{2}$ NYU Hospital for Joint Disease, Rheumatology, New YorK, United States of America; ${ }^{3}$ Istanbul University-Cerrahpasa, Paediatric Rheumatology, Instanbul, Turkey; ${ }^{4}$ Dokuz Eylul University, Paediatric Rheumatology, Izmir, Turkey; ${ }^{5}$ Hacettepe University, Ankara, Paediatric Rheumatology, Ankara, Turkey; ${ }^{6}$ New York University School of Medicine, Rheumatology, New York, United States of America

Background: Behçet disease (BD) is a systemic inflammatory disease which is rare in children. It is also known a higher prevalence along the ancient Silk Road and the eastern Mediterranean, especially in Turkey. Despite the presence of diagnostic criteria, the diagnosis of pediatric BD is still difficult due to atypical findings and the heterogeneity of the disease. While descriptive cohort studies in pediatric BD exist, direct comparison studies of clinical manifestations and disease activity between patients from different countries are scarce.

Objectives: We aimed to compare the main clinical features and activity of pediatric BD patients from Turkey versus United States (US).

Methods: The BD was diagnosed before 18 years of age and based on expert opinion. Disease activity was assessed with Physician Global Assessment (PhGA), Parents/Patient Global Assessment (PWPGA) and Behçet's Syndrome Activity Scale (BSAS) were administered to patients from both countries.

Results: A total of 161 patients were included (61 from Turkey; 100 from US). Males were more prevalent among patients from Turkey than among patients from US $(63.9 \%$ vs. $32 \%$, respectively, $p<0.001)$. Disease duration at the diagnosis was significantly longer in US $p=0.002$ ). Oral aphthosis was the most common symptom in both groups (96.7\% for Turkey and $73 \%$ for US), however a significant difference was found $(p<0.001)$. Genital ulcers were documented in $47 \%$ of US patients and in $41.4 \%$ of Turkish patients $(p=0.5)$. There were significant difference between two groups with regards to the ocular involvement: uveitis and posterior uveitis were more frequently in Turkish patients $(p=<0.001$ respectively) while retinal vasculitis was no seen in US patients $(p<0.001)$. Erythema nodosum occurred more frequently in Turkish group $(p<0.001)$. The pathergy test was reported as positive in 22 $(37.3 \%)$ patients in Turkey and $2(2.3 \%)$ in USA in our study $(p<0.001)$. Gastrointestinal and neurological involvement did not differ between cohorts. The rate of colchicine and oral steroids was similar. Azathioprine, cyclosporine and methotrexate usage was more frequent in Turkey $(p=0.005, p=0.007$, $p=0.033$, respectively) and infliximab was administered just in the US cohort $(p=0.002)$. PhGA and BSAS scores were higher in patients from Turkey $(p=0.003$ and $p=0.017$ respectively) and no significant differences were seen in PWPGA scores.

Conclusion: Disease activity and clinical features seem to be different between the two countries which may be linked to the environmental factors, referra patterns and immune system responses in the expression of this disease. The diagnosis of BD in the US was based on both fulfilling the ISBD criteria and in cases when criteria were not met, based on treating physician assessment which can explain some of the differences in disease presentation. In addition, there were differences in regard to clinical practice and treatment patterns between two countries. Increasing knowledge about heterogeneity of BD will improve the ability of diagnosis, development of new diagnostic criteria, and management of $\mathrm{BD}$.

\section{REFERENCES:}

[1] Butbul Aviel Y et al, Semin Arthritis Rheum. 2020

Disclosure of Interests: None declared

DOI: 10.1136/annrheumdis-2021-eular.3132 